\title{
Correction to: Nonlinear deformation of tractography in ultrasound-guided low-grade gliomas resection
}

\author{
Yiming Xiao ${ }^{1,2}$ (D) $\cdot$ Live Eikenes $^{3} \cdot$ Ingerid Reinertsen $^{4,5}$ \\ Hassan Rivaz ${ }^{1,2}$ \\ Published online: 1 February 2018 \\ (c) CARS 2018

\section{Correction to:}

$$
\begin{aligned}
& \text { International Journal of Computer } \\
& \text { Assisted Radiology and Surgery } \\
& \text { https://doi.org/10.1007/s11548-017-1699-x }
\end{aligned}
$$

The author would like to include grant number of NSERC Discovery Grant in the acknowledgement section of the original article.

Acknowledgement section should read as:

This project was partly funded by NSERC Discovery Grant (RGPIN-2015-04136) and the Norwegian National Advisory Unit for Ultrasound and Image-Guided Therapy. The authors thank Dr. Maryse Fortin for her generous help in identifying the anatomical landmarks.

The original article can be found online at https://doi.org/10.1007/ s11548-017-1699-x.

Yiming Xiao

yiming.xiao@concordia.ca

1 PERFORM Centre, Concordia University, Rm 2.211, 7200 Sherbrooke St. W., Montreal, QC H4B 1R6, Canada

2 Department of Electrical and Computer Engineering, Concordia University, Montreal, Canada

3 Department of Circulation and Medical Imaging, Norwegian University of Science and Technology, Trondheim, Norway

4 Department of Medical Technology, SINTEF, Trondheim, Norway

5 Norwegian National Advisory Unit for Ultrasound and Image-Guided Therapy, St. Olavs University Hospital, Trondheim, Norway 